\title{
A genetic variation in the PGC-1 gene could confer insulin resistance and susceptibility to Type II diabetes
}

\author{
K. Hara ${ }^{1,2,3}$, K. Tobe ${ }^{1,2}$, T. Okada ${ }^{1,4}$, H. Kadowaki ${ }^{3}$, Y. Akanuma ${ }^{2,3}$, C. Ito ${ }^{5}$, S. Kimura ${ }^{1}$, T. Kadowaki ${ }^{1,2}$ \\ ${ }^{1}$ Department of Metabolic Diseases, Graduate School of Medicine, University of Tokyo, Hongo 7-3-1, Bunkyo-ku, \\ Tokyo 113-8655, Japan \\ ${ }^{2}$ CREST, Japan Science and Technology Corporation (JST), Chuo-ku, Tokyo, Japan \\ 3 The Institute for Diabetes Care and Research, Asahi Life Foundation, Tokyo, Japan \\ ${ }^{4}$ Department of Metabolic Disorder, Research Institute, International Medical Center of Japan, Tokyo, Japan \\ ${ }^{5}$ Hiroshima Atomic Bomb Casualty Council Health Management Center, Hiroshima, Japan
}

\section{Abstract}

Aims/hypothesis. Peroxisome proliferator activated receptor $\gamma$ coactivator-1 (PGC-1), a transcriptional coactivator of the nuclear receptor PPAR $\gamma$, plays a role in adaptive thermogenesis and insulin sensitivity. Plasma fasting insulin has been linked to the chromosomal region where the $P G C-1$ gene is located. Thus, $P G C-1$ can be viewed as a functional and positional candidate for the susceptibility gene for Type II (non-insulindependent) diabetes mellitus.

Methods. After screening the $P G C-1$ gene for single nucleotide polymorphisms (SNPs), we performed an association study using the newly detected SNPs in 537 Type II diabetic patients and 417 non-diabetic subjects.

Results. We found three relatively frequent SNPs in the PGC-1 gene (IVS4-11T >C, Thr394Thr and
Gly482Ser). There were significant differences in fasting insulin (Gly/Gly; $37.7 \pm 1.43$, Gly/Ser; $40.2 \pm$ 1.21 , Ser/Ser; $44.3 \pm 1.82 \mathrm{pmol} / 1, p=0.018)$ and insulin resistance index (Gly/Gly; $1.48 \pm 0.06$, Gly/Ser; $1.56 \pm 0.05$, Ser/Ser; $1.75 \pm 0.08, p=0.027$ ) according to the genotype of the Gly482Ser polymorphism. The Thr394Thr - Gly482Ser haplotype was associated with Type II diabetes $(p=0.00003)$.

Conclusion/interpretation. The results of this study suggested that the $P G C-1$ gene might be implicated in the pathogenesis of Type II diabetes. [Diabetologia (2002) 45:740-743]

Keywords Single nucleotide polymorphism, HOMA, transcriptional coactivator, susceptibility gene, association study, direct sequencing, linkage disequilibrium, haplotype, PCR-RFLP.
A co-activator of nuclear receptors, $P G C-1$ (peroxisome proliferator activated receptor $\gamma$ coactivator-1), has been cloned from a brown fat cDNA library [1].

Received: 22 October 2001 / Revised: 31 December 2001 Published online: 23 April 2002

(C) Springer-Verlag 2002

Corresponding author: T. Kadowaki, Department of Metabolic Diseases, Graduate School of Medicine, University of Tokyo, Hongo 7-3-1, Bunkyo-ku, Tokyo 113-8655, Japan, E-mail: kadowaki-3im@h.u-tokyo.ac.jp

Abbreviations: PGC-1, Peroxisome proliferator activated receptor gamma coactivator-1; PPAR, peroxisome proliferator activated receptor; SNP, single nucleotide polymorphism; UCP, uncoupling protein; GLUT, glucose transporter; QTL, quantitative-trait linkage; HOMA, homeostasis model assessment
$P G C-1$ is thought to play a role in energy homeostasis, especially in adaptive thermogenesis. Substantial differences in responses to a caloric overload among individuals have been reported [2] and reduced adaptive thermogenesis leads to obesity especially under certain circumstances, such as immobility or a highfat diet [3]. Thus, alteration in the function or expression of PGC-1 could lead to changes in adiposity.

PGC-1 has a role in regulating expression of GLUT4 in skeletal muscle [4] and has an effect on regulation of gluconeogenesis in the liver [5], making $P G C-1$ an attractive candidate for a susceptibility gene for obesity or Type II diabetes or both.

This hypothesis has been supported by a quantitative-trait linkage (QTL) analysis in Pima Indians that suggests linkage of basal insulin concentrations, an indicator of insulin sensitivity, to chromosome 4 p15.1 
[6], where the $P G C-1$ gene is located [7]. Therefore, we studied whether genetic variations in the $P G C-1$ gene might influence insulin resistance and susceptibility to Type II diabetes.

\section{Subjects and methods}

Subjects. Non-diabetic subjects over 60 years of age who had $\mathrm{HbA}_{1 \mathrm{c}}$ values below $5.8 \%$ and no family history of Type II diabetes were recruited from the Hiroshima Atomic Bomb Casualty Council Health Management Center in Tokyo, Japan. The diabetic subjects were randomly recruited from the Department of Metabolic Diseases, University of Tokyo. Diabetes was diagnosed according to the World Health Organisation (WHO) criteria [8] and 417 Type II diabetic and 537 non-diabetic subjects were enrolled in the present study. All the subjects enrolled in this study were of full Japanese ethnicity. The study was performed after obtaining the informed consent of all the subjects and was approved by the Ethics Committee of the University of Tokyo.

Biological measurements. Insulin resistance and beta-cell function were assessed by homeostasis model assessment (HOMA) (homeostasis model assessment of insulin resistance (HOMAIR) $=$ fasting insulin $(\mu \mathrm{U} / \mathrm{ml}) \times$ glucose $(\mathrm{mmol} / \mathrm{l}) / 22.5$, betacell function $($ HOMA-beta) $=20 \times$ fasting insulin $(\mu \mathrm{U} / \mathrm{ml}) /($ fast ing glucose (mmol/l)-3.5)), as described elsewhere [9].

Screening for SNPs in the PGC-1 gene. The coding region of all exons of the $P G C-1$ gene and their flanking regions was screened in 18 Type II diabetic patients and 18 non-diabetic subjects, based on the sequence reported in GenBank (accession number AF108193- AF108205). The conditions and sequences of the primers used in the PCR are available from the author.

Genotyping of SNPs detected. The three relatively frequent polymorphisms were genotyped by the polymerase chain reaction-restriction fragment length polymorphism (PCR-RFLP) method. The sequences of the primers to detect Gly482Ser polymorphism were 5'-TTTGGAGGCAAGCAAGCAG-3' and 5'-TATTTAGGGTTTTGCCAAGG-3'. The sequences of the primers to detect intron 4 polymorphism were $5^{\prime}$-TTCAGTTCACTAATTTTAGCT-TTCTTTAGGCC-3' containing one nucleotide mismatch (underlined), which made it possible to use the restriction enzyme Msp I (New England Biolabs, Beverly, Mass., USA) for detection of intron 4 polymorphism and 5'-TCCCTCACCAACAGCTCGT-3'. The sequences of the primers to detect $\mathrm{Thr} 394 \mathrm{Thr}(\mathrm{ACG} \rightarrow \mathrm{ACA})$ polymorphism were 5'-GCCAGTCAATTAA-TTCCAAACC-3' containing one nucleotide mismatch (underlined), which made it possible to use the restriction enzyme Msp I (New England Biolabs, Beverly, Mass., USA) and 5'-TTGGAGCTGTTTTCTTGTGC3'. PCR was carried out using 1.25U of Ampli Taq Gold (Applied Biosystems, Foster City, Calif., USA) with annealing at $57^{\circ} \mathrm{C}\left(\right.$ IVS4-11T > C), $53^{\circ} \mathrm{C}$ (the Thr394Thr), or $52^{\circ} \mathrm{C}$ (the Gly482Ser) for $30 \mathrm{~s}$. PCR products were digested overnight at $37^{\circ} \mathrm{C}$ with Msp I for the IVS4-11T >C and the Thr394Thr, and with Stu I for the Gly482Ser, and the products were resolved by $3 \%$ ethidium bromide-stained agarose gel electrophoresis.

Statistical analysis. The proportions of genotypes or alleles were compared by the chi square $\left(\chi^{2}\right)$ test. Frequencies of haplotypes were estimated by using the Estimation Haplotypefrequencies software (EH) software (ftp://linkage.rockefel- ler.edu/software/eh). The probability of having those estimated distributions of haplotypes in Type II diabetic (Ld) and nondiabetic (Ln) subjects was compared with that in the combined subjects (Lall). The degree of statistical significance was evaluated assuming that the likelihood ratio, [Lall-(Ln+Ld)], is distributed as a $\chi^{2}$ with degree of freedom being three. The statistical analyses except haplotype analysis were performed using SAS for WINDOWS ver 6.12 software (SAS Institute, Cary, N.C., USA). The pair-wise linkage disequilibrium parameter $D^{\prime}$ was calculated as previously described [10].

\section{Results}

Scanning of the coding regions of the $P G C-1$ gene and their flanking regions yielded three relatively frequent SNPs IVS4-11T >C, Thr394Thr, and Gly482Ser. The genotypic distributions of the three polymorphisms were in Hardy-Weinberg equilibrium both in the non-diabetic subjects and in the Type II diabetic subjects.

We investigated differences in insulin sensitivity and insulin secretion according to the SNPs in the $P G C-1$ gene by homeostasis model assessment [8] in the non-diabetic subjects. Fasting insulin (Gly/Gly; $37.7 \pm 1.43$, Gly/Ser; $40.2 \pm 1.21$, Ser/Ser; $44.3 \pm 1.82$ $\mathrm{pmol} / \mathrm{l}, \quad p=0.018)$ and insulin resistance index (Gly/Gly; $1.48 \pm 0.06$, Gly/Ser; $1.56 \pm 0.05$, Ser/Ser; $1.75 \pm 0.08, p=0.027$ ) differed significantly according to the genotypes of the Gly482Ser polymorphism after adjustment for age, gender, and BMI (Table 1). Because we studied insulin resistance only in the nondiabetic subjects in this study, we believe that estimation of insulin resistance by HOMA index is acceptable as a convenient approximation. Nevertheless, interpretation of the results using HOMA index should be made with caution until these results are confirmed by euglycaemic hyperinsulinaemic clamp. There were no differences in genotype or allele distribution between the Type II diabetic and non-diabetic subjects according to each SNP. The Thr394Thr and Gly482 Ser polymorphisms were in strong, but not in complete, linkage disequilibrium $\left(D^{\prime}=0.86\right)$ with each other. Comparison of the frequencies of the Thr394ThrGly482Ser haplotypes in the Type II diabetic subjects and the non-diabetic subjects revealed significantly different distribution of the haplotypes between Type II diabetic and non-diabetic subjects $(p=0.00003)$ (Table 2). Given the large sample sizes we studied, the estimated haplotype frequencies are not likely to deviate from the true values. However, because error resulting from estimation of haplotype frequencies was not incorporated into the test, this $p$ value is tentative. Because no significant association between individual SNPs and Type II diabetes was found, the haplotype analysis indicates that an unidentified SNP which is in linkage disequilibrium with this haplotype and is related to the pathogenesis of Type II diabetes could exist. Furthermore, the haplotype itself could have some role in the development of Type II diabetes. 


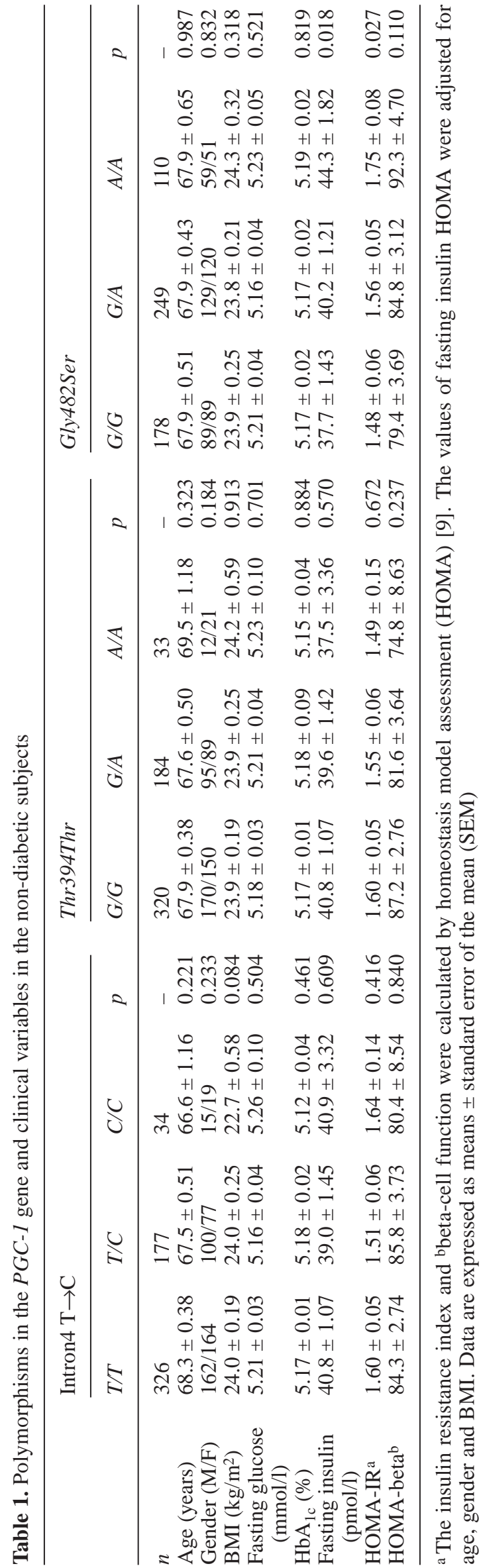

Table 2. Distributions of Thr394Thr Gly482Ser haplotypes in Type II diabetic and non-diabetic subjects

\begin{tabular}{lll}
\hline Haplotype & $\begin{array}{l}\text { Type II diabetic } \\
\text { subjects }\end{array}$ & $\begin{array}{l}\text { Non-diabetic } \\
\text { subjects }\end{array}$ \\
\cline { 2 - 3 } & $n(\%)$ & $n(\%)$ \\
\hline Thr394 (ACG) - Gly482 & $302(36.2)$ & $368(34.3)$ \\
Thr394 (ACG) - Ser482 & $351(42.1)$ & $454(42.2)$ \\
Thr394 (ACA) - Gly482 & $143(17.1)$ & $238(22.2)$ \\
Thr394 (ACA) - Ser482 & $38(4.6)$ & $14(1.3)$ \\
\hline
\end{tabular}

There was a significant difference in the distribution of Thr394Thr (ACG $\rightarrow$ ACA) - Gly482Ser haplotypes between Type II diabetic and non-diabetic subjects $(p=0.00003)$

\section{Discussion}

It has been reported that $P G C-1$ has a role in regulating expression of GLUT4 in skeletal muscle [4] and also an effect on the regulation of gluconeogenesis in the liver [5]. Thus, reduction in the activity of the $P G C$ - 1 gene might lead to the insulin resistance in muscle and the opposite effect in the liver, and a functional study, such as gene-targeting in mice, is needed to clarify the role of PGC-1 as a whole.

In summary, a genetic variation in the $P G C-1$ gene was found to be associated with insulin resistance. The Thr394Thr - Gly482Ser haplotype was significantly associated with Type II diabetes, although individual SNPs were not. Taken together with the reported data showing that plasma fasting insulin is linked to chromosome $4 \mathrm{p} 15.1$ [6], where the $P G C-1$ gene is located [7], the results of the study suggest that the $P G C-1$ gene is implicated in the pathogenesis of Type II diabetes.

Acknowledgements. This work was supported by grant-in-aid for the development of innovative technology from the Ministry of Education, Culture, Sports, Science and Technology (to T. Kadowaki), by grant-in aid for Creative Scientific Research 10NP0201 from Japan Society for the Promotion of Science (to T. Kadowaki), by Health Science Research Grants (Research on Human Genome and Gene Therapy) from the Ministry of Health and Welfare (to T. Kadowaki), and by grant-inaid for Scientific Research on Priority Areas (C) to $\mathrm{T}$. Kadowaki. We thank Ms M. Yunokizaki and Ms Y. Okada for technical assistance.

\section{References}

1. Puigserver P, Wu Z, Park CW, Graves R, Wright M, Spiegelman BM (1998) A cold-inducible coactivator of nuclear receptors linked to adaptive thermogenesis. Cell 92:829-839

2. Bouchard C, Tremblay A, Despres JP et al. (1990) The response to long-term overfeeding in identical twins. N Engl J Med 322:1477-1482

3. Levine JA, Eberhardt NL, Jensen MD (1999) Role of nonexercise activity thermogenesis in resistance to fat gain in humans. Science 283:212-214 
4. Michael LF, Wu Z, Cheatham RB et al. (2001) Restoration of insulin-sensitive glucose transporter (GLUT4) gene expression in muscle cells by the transcriptional coactivator $P G C$-1. Proc Natl Acad Sci USA 98:3820-3825

5. Yoon JC, Puigserver P, Chen G et al. (2001) Control of hepatic gluconeogenesis through the transcriptional coactivator PGC-1. Nature 413:131-138

6. Pratley RE, Thompson DB, Prochazka M et al. (1998) An autosomal genomic scan for loci linked to prediabetic phenotypes in Pima Indians J Clin Invest 101:1757-1764

7. Esterbauer H, Oberkofler H, Krempler F, Patsch W (1999) Human peroxisomal proliferator activated receptor gamma coactivator 1 (PPARGC1) gene: cDNA sequence, genomic organization, chromosomal localization, and tissue expression. Genomics 62:98-102
8. Alberti KGMM, Zimmet PZ (1998) Definition, diagnosis and classification of diabetes mellitus and its complications part 1: Diagnosis and classification of diabetes mellitus. Provisional report of a WHO consultation. Diabet Med 15:539-553

9. Matthews DR, Rudenski AS, Naylor BA, Treacher DF, Turner RC (1985) Homeostasis model assessment. Insulin resistance and beta-cell function from fasting plasma glucose and insulin concentrations in man. Diabetologia 28:412-419

10. Jorde LB, Watkins WS, Carlson M et al. (1994) Linkage disequilibrium predicts physical distance in the adenomatous polyposis coli region. Am J Hum Genet 54:884-898 